\title{
Rate-Constrained Adaptive Quantization Scheme for Wavelet Pyramid Image Coding
}

\author{
J.Q. Ni \\ jqni@hkueee.hku.hk \\ K.L. Ho \\ klho@hkueee.hku.hk \\ K.W. Tse \\ kwtse@hkueee.hku.hk \\ Department of Electrical and Electronic Engineering \\ The University of Hong Kong \\ Pokfulam Road, Hong Kong
}

\begin{abstract}
It is well known that orthogonal wavelet transform with filters of nonlinear phase gives poor visual results in low bit rate image coding. Biorthogonal wavelet is a good substitute, which is, however essentially nonorthogonal. A greedy steepest descend algorithm is proposed to design an adaptive quantization scheme based on the actual statistics of the input image. Since the $L^{2}$ norm of the quantization error is not preserved through the nonorthogonal transform, a quantization error estimation formula considering the characteristic value of the reconstruction filters is derived to incorporate the adaptive quantization scheme. Computer simulation results demonstrate significant SNR gains over standard coding technique, and comparable visual improvements.
\end{abstract}

\section{Introduction}

Recently wavelet based image coding has become the domain of extensive research. For image coding application, it is desirable that an exact reconstruction subband coding scheme should correspond to an orthonormal basis with reasonable smooth mother wavelet. However, the resulting analysis and synthesis filters are in general not linear phase. On the other hand it is also desirable the FIR filter used be linear phase, since such filter can be easily cascaded in pyramidal filter structure without phase compensation [2]. Unfortunately, there are no nontrivial orthonormal linear phase FIR filters with the exact reconstruction property [5]. Relaxing the orthonormality requirement and using biorthogonal basis is a good alternative.

As is well known, the $L^{2}$ norm of quantization errors is not preserved through the nonorthogonal transform, so there does not exist an exact analytical formula for optimal bit allocation as in the case of orthogonal wavelet transform [3]. The goal of the paper is to improve the applicability of biorthogonal wavelet transform to image coding by proposing an adaptive quantization design scheme based on the statistics of the input image. The design procedure is based on a criterion which best describes the trade-off between distortion and rate, and hence results in superior performance.

\section{Biorthogonal Wavelets}

\section{II.1 Siganl Analysis with Biorthogonal Wavelet}

Biorthogonal wavelet bases were firstly introduced by Cohen [5] and were extensively used by numerous authors in image coding application. The basic idea of biorthogonal wavelet transform is to represent a signal $f(x)$ as a superposition of two biorthogonal bases

$$
\psi_{m, n}(x)=2^{-m / 2} \psi\left(2^{-m} x-n\right)
$$

and $\psi_{m, n}^{*}(x)=2^{-m / 2} \psi^{*}\left(2^{-m / 2} x-n\right)$, where $\psi^{*}(x)$

is the dual basis of $\psi(x), \mathrm{m}$ and $\mathrm{n}$ are scaling and translation parameters respectively. Therefore the signal $f(x)$ can be expressed as

$$
f=\sum_{m, n} c_{m, n}(f) \psi_{m, n}^{*}
$$

and

$$
c_{m, n}(f)=\left\langle\psi_{m, n}, f\right\rangle=\int_{-\infty}^{+\infty} \psi_{m, n}(x) f(x) d x
$$

As in the orthonormal case, one can introduce multiresolution analysis. We then define two dual scaling functions: $\phi_{m, n}(x)=2^{-m / 2} \phi\left(2^{-m} x-n\right)$ and its dual $\phi_{m, n}^{*}$.

A pair of filter $h$ and $h^{*}$ have been introduced for decomposition and reconstruction of signal $f$ to and from its wavelet representation respectively. Generally speaking, there are two pairs of filters: one pair $h$ and $g$ for decomposition and another pair $h^{*}$ and $g^{*}$ for reconstruction. Here only $h$ and $h^{*}$ are independent. The relations between the different filters are given by the following formulas [2]:

$$
\begin{gathered}
g_{n}^{*}=(-1)^{n} h_{1-n} \text { and } g_{n}=(-1)^{n} h_{1-n}^{*} \\
\sum_{n} h_{n} h_{n+2 k}=\delta_{k, 0}
\end{gathered}
$$

which ensures perfect reconstruction ( PR ).

For signal decomposition, we have

$$
\begin{aligned}
& c_{m, n}=\sum_{k} g_{2 n-k} a_{m-1, k} \\
& a_{m, n}=\sum_{k} h_{2 n-k} a_{m-1, k}
\end{aligned}
$$


while for signal reconstruction,

$$
a_{m-1, n}=2 \times \sum_{k}\left(h_{2 k-n}^{*} a_{m, k}+g_{2 k-n}^{*} c_{m, k}\right)
$$

II. 2 Extension to the 2-D case

The results described in the previous section can be easily extended to 2-dimensional case by applying two 1$D$ wavelet transform separately [2].

In 2-D dyadic wavelet analysis, as in the 1-D case, we introduce a scaling function $\phi(x, y)$ such that:

$$
\phi(x, y)=\phi(x) \phi(y)
$$

where $\phi(x)$ is a one-dimensional scaling function.

Let $\psi(x)$ denotes the one-dimensional wavelet associated with the scaling functionm $\phi(x)$. Then, we have three two-dimensional wavelets defined as follows:

$$
\begin{aligned}
& \psi^{H}(x, y)=\phi(x) \psi(y) \\
& \psi^{V}(x, y)=\psi(x) \phi(y) \\
& \psi^{D}(x, y)=\psi(x) \psi(y)
\end{aligned}
$$

\section{II.3 Image Coding with Biorthogonal Wavelets}

A wide variety of wavelet-based image compression schemes have been reported in previous literatures, ranging from simple entropy coding to more complex techniques such as vector quantization [2], adaptive transforms, embedded zerotree encoding [7] and edgebased coding [5]. The principle of wavelet based image coding can be described as follows: the input image $P_{0}$ is fed into the first WT unit, the "smoothed" version $P_{1}$ is again fed into next WT unit for further decomposition while the wavelet details $D_{1}^{1}, D_{1}^{2}$ and $D_{1}^{3}$ are sent to a buffer for quantizing and encoding. This procedure is repeated $J$ times, forming a $J$ layer pyramid structure $\left\{P_{J},\left(D_{j}^{1}\right)_{1 \leq j \leq J},\left(D_{j}^{2}\right)_{1 \leq j \leq J},\left(D_{j}^{3}\right)_{1 \leq j \leq J}\right\}$, which is called the wavelet pyramid. The "information packing" property of wavelet transform makes the image in wavelet domain have a lower entropy than the original, thereby increasing the possibility of compression.

\section{Adaptive Quantization Scheme for Image Pyramid Coding}

\section{III.1 Problem Formulation}

The proposed adaptive quantization scheme is a scalar quantizer. If a variable-length entropy encoder is used, uniform quantization is optimal. To achieve the best results, a separate quantizer should be designed for each subband, which is equivalent to determine the optimal step size $\Delta$ for each subband based on the statistics of input image. If we represent the original image with $\mathrm{J}$ level wavelet pyramid, for a predefined target bit rate $R_{T \text { arget }}$, the problem is formulated as to find a set of optimal step size $\left\{\Delta_{J},\left(\Delta_{j}^{k}\right)_{1 \leq j \leq J, 1 \leq k \leq 3}\right\}$ to minimize the overall distortion

$$
\varepsilon=D\left(\Delta_{J}, \Delta_{j}^{k}\right)
$$

subject to the target bit rate constraint:

$$
\left(\frac{1}{4^{J}} R_{J}+\sum_{j=1}^{J} \frac{1}{4^{j}} \sum_{k=1}^{3} R_{j}^{k}\right) \leq R_{T \text { arget }}
$$

Since $L^{2}$ norm is not preserved through biorthorgonal wavelet transform, the $\varepsilon$ has not the simple form of direct summation of quantization error of each subband as in the orthogonal case. To estimate the overall distortion $\varepsilon$, it is not necessary to perform the complete inverse wavelet transform each time the set of quantization step is updated. In subband image coding, one can assume that quantization error generated in one subband will not influence the error generation in other subband during reconstruction [4]. Therefore the overall distortion depends upon the frequency characteristic of reconstruction filters. Then the average distortion per sample after reconstruction can be expressed as [4] :

$$
\sigma_{n}^{2}=\gamma_{l} \sigma_{n l}^{2}+\gamma_{h} \sigma_{n h}^{2}
$$

where $\sigma_{n l}^{2}, \sigma_{n h}^{2}$ and $\gamma_{l}$ and $\gamma_{h}$ are the quantization error and characteristic value of reconstruction filter corresponding to low-pass and high-pass subbands, respectively. And $\gamma_{l}, \gamma_{h}$ have the form (for normalized filter pair $h$ and $h^{*}$ ):

$$
\begin{aligned}
& \gamma_{l}=\frac{1}{\pi} \int_{-\pi}^{\pi}\left|H^{*}(\omega)\right|^{2} d \omega \\
& \gamma_{h}=\frac{1}{\pi} \int_{-\pi}^{\pi}\left|G^{*}(\omega)\right|^{2} d \omega
\end{aligned}
$$

The extension of above results to 2-dimensional case is straightforward. Let $\gamma_{0}$ denote the characteristic value of reconstruction filter for LL band, $\gamma_{1}$ for LH band, $\gamma_{2}$ for $\mathrm{HL}$ band and $\gamma_{3}$ for $\mathrm{HH}$ band, for a J-layer pyramid image decomposition, we have

$$
\varepsilon=\gamma_{0}^{J} \varepsilon_{J}+\sum_{j=1}^{J} \sum_{k=1}^{3} \gamma_{0}^{j-1} \gamma_{k} \varepsilon_{j}^{k}
$$

where $\gamma_{0}=\gamma_{l} \gamma_{l}, \quad \gamma_{1}=\gamma_{l} \gamma_{h}, \quad \gamma_{2}=\gamma_{h} \gamma_{l}$ and $\gamma_{3}=\gamma_{h} \gamma_{h}$

Rate estimation is another key issue involved in the quantizer design procedure. We do not restrict ourselves to a particular entropy encoder for the sake of generality, instead we adopt an empirical entropy $R_{T}$ introduced by Antonini [2]:

$$
R_{T}=\frac{1}{4^{J}} R_{J}+\sum_{j=1}^{J} \frac{1}{4^{j}} \sum_{k=1}^{3} R_{j}^{k}
$$


where $R_{J}$ and $R_{j}^{k}$ represent the bit rates assigned to sub-image $P_{J}$ and $P_{j}^{k}$, respectively. For sub-image $P_{j}^{k}$, we can estimate the average entropy as follows:

$$
\mathfrak{R}_{j}^{k}=-\frac{1}{4^{j}} \sum_{i=1}^{L} p\left(w_{i}\right) \log _{2} p\left(w_{i}\right)
$$

$w_{i}$ in above expression denotes the codeword corresponding to $P_{j}^{k}$.

\section{III.2 The Adaptive Quantization Scheme}

The proposed adaptive quantization scheme follows its spirit from Wu's algorithm [1] for JPEG baseline coders with some modification. It is felt that the proposed scheme is more suitable for wavelet-based image coding application. Compared to the quantization table in JPEG baseline coders, the number of entry in the set of quantization step, which equals to the number of subbands in wavelet pyramid, is greatly reduced, therefore the complexity of the algorithm is greatly reduced.

The basic concept of the scheme is described as follows: starting from a set of initial large quantization step $\left\{\Delta_{J},\left(\Delta_{j}^{k}\right)_{1 \leq j \leq J, 1 \leq k \leq 3}\right\}$ for each subband, corresponding to low bit rates and high reconstruction distortion, the algorithm decreases the step size of one entry of the quantization set at a time until a predefined target bit rate is reached. A reasonable quantity that best describes the rate-distortion trade-off is $\frac{-\Delta \varepsilon}{\Delta R}$, i.e., the ratio between the decrease in reconstruction error and the increase in bit rates. We re-lable the components of quantization table $\Delta=\left\{\Delta_{J},\left(\Delta_{j}^{k}\right)_{1 \leq j \leq J, 1 \leq k \leq 3}\right\} \quad$ to give $\Delta=\left\{\Delta_{0}, \Delta_{1}, \cdots, \Delta_{3 J}\right\}$ for convenient description of the scheme. Then we can describe the procedure of quantizer design as the following optimization problem:

$$
\left.\max _{k} \max _{\bar{\Delta}_{k}} \frac{-\Delta \varepsilon}{\Delta R}\right|_{\Delta_{k}} \rightarrow \bar{\Delta}_{k}
$$

The optimization problem of ( 15 ) can be divided into two parts; the first part calculates

$$
\lambda_{k}=\left.\max _{\bar{\Delta}_{k}} \frac{-\Delta \varepsilon}{\Delta R}\right|_{\Delta_{k}} \rightarrow \bar{\Delta}_{k}
$$

for all $\mathrm{k}$ and the second part finds

$$
\lambda=\max _{k} \lambda_{k}
$$

We set the initial value of $\Delta_{0}$ in quantization table $\left\{\Delta_{k}\right\}$ as some small value, e.g. 16 , to preserve the visual quality of image. The algorithm also maintains two extra tables $\left\{\lambda_{k}\right\}$ and $\left\{\Delta_{k}^{*}\right\}$ where the $\Delta_{k}^{*}$ is the solution for
(16-1). The design algorithm is iterative, and is given as follows:

1) Initially set the quantization table $\left\{\Delta_{k}\right\}$ by $\Delta_{0}=$ some small step; $\Delta_{k}=\Delta_{\max }$ for $k=1, \cdots, 3 J$

2) Initialize the tables of $\left\{\lambda_{k}\right\}$ and $\left\{\Delta_{k}^{*}\right\}$ by searching $\Delta_{k} \quad$ within domain $\quad\left\{1, \cdots, \Delta_{\max }-1\right\}$ for $k=1, \cdots, 3 J$ and $\left\{1, \cdots, \Delta_{0}-1\right\}$ for $\mathrm{k}=0$ so that $(16-1)$ has the maximum.

3) Search $\mathrm{k}$ in $\{0,1, \cdots, 3 J\}$ for $k^{*}$ to solve (16-2).

4) Update $k^{*}$-th entry of the quantization table $\left\{\Delta_{k}\right\}$ by setting $\Delta_{k^{*}}=\Delta_{k^{*}}^{*}$

5) Update $\lambda_{k^{*}}$ and $\Delta_{k^{*}}^{*}$ by searching $\Delta$ in $\left\{1, \cdots, \Delta_{k^{*}}^{*}-1\right\}$ to solve (16-1) for $k=k^{*}$.

6) if $R_{T} \geq R_{T}$ arget then stop,else goto 3 .

The design algorithm terminates when the bit rate equal or exceeds the target bit rate $R_{T \text { arget }}$. For normalized filter pair $h(n)$ and $h^{*}(n)$, the absolute value of WT coefficient ranges from 0 to 255 and the $\Delta_{\max }$ can be set to 255. However, computer simulation suggests that, as stated in Wu'paper, for practical image coding application at a reasonable bit rate, initializing the quantization step table with some relatively small step size leads almost the same result as with the maximum allowable step size 255 , but the number of iteration required to arrive at the target bit rate is greatly reduced.

\section{Simulation Result}

Computer simulation was carried out to verify the feasibility and effectiveness of the proposed adaptive quantization design scheme for wavelet pyramid image coding. We compare the performance between the conventional and adaptive quantization scheme with the standard test image "Lena" of size $512 \times 512$. In both cases the test image is decomposed with the same nine and seven taps biorthogonal wavelet [2]. The conventional quantization scheme uses the same step size for each band at the same resolution, decreasing by a factor of 2 when going to next coarser resolution, which is optimal for orthogonal wavelet decomposition [3]. We obtain PSNR of $34.5 \mathrm{db}$ at $0.4 \mathrm{bbp}$ and $32.3 \mathrm{db}$ at $0.2 \mathrm{bbp}$ with the proposed adaptive quantization scheme, compared to 32.2 at $0.4 \mathrm{bbp}$ and $30.1 \mathrm{db}$ at $0.2 \mathrm{bbp}$ with conventional quantization scheme. Fig. 1 gives the original test image and the reconstructed images with the proposed scheme at different bit rates. Significant improvement over conventional quantization scheme is observed both in PSNR perfomance and overall visual quality of coded image at the same bit rate. 


\section{Conclusion}

In this paper, we have presented a rate-constrained adaptive quantization scheme for wavelet based pyramid image coding. The scheme is based on a criterion which gives the best trade-off between distortion and bit rate and adapts to the actual statistics of the input image. The use of biorthogonal wavelet leads to better visual quality of coded image, which is, however, nonorthogonal in nature and the $L^{2}$ norm of the quantization error is not preserved through nonorthogonal transform. To incorporate the adaptive quantization scheme, we have derived the formula of quantization error estimation, considering the characteristic value of reconstruction filter, for biorthogonal wavelet transform under the assumption of cross-uncorrelated quantization error among subbands, which seems to work well for most working wavelets. Empirical entropy is also used to evaluate the bit rate of the coded image when the adaptive quantization scheme is applied to the decomposed image. The immediate estimation of both reconstruction error and bit rate makes the proposed adaptive scheme feasible and fast in implementation. Computer simulation shows considerable SNR gains over conventional scheme and comparable visual improvement.

\section{Reference}

[1] S.W. Wu and A. Gersho, "Rate-Constrainted PictureAdaptive Quantization for JPEG Baseline Coders," in Proc . Inter. Conf. on ASSP, pp. 365-368, April,1993.

[2] M. Antonini, M. Barlaud, M. Mathieu and Daubechies, "Image Compression Using Wavelet Transform," IEEE Trans. Image Processing, Vol.1, no.2, pp. 205-220,1992.

[3] J. Chen, S. Itoh and T. Hashimoto, "Scalar Quantization Noise Analysis and Optimal Bit Allocation for Wavelet Pyramid Image Coding," IEICE Trans. Fundamentals, Vol. E76-A, pp. 1502-1514, Sep. 1993.

[4] C.K. Cheong, K. Aizawa, T. Saito and M. Hatori, "Subband Image Coding with Biorthogonal Wavelets," IEICE Trans. Fundamentals, Vol. E75-A, pp. 871-881, July 1992.

[5] C.K. Chui, Wavelets: A Tutorial in Theory and applications, Academic Press,Inc.,New York,1992.

[6] S. Mallat, "A Theory for Multiresolution Signal Decomposition: The Wavelet Representation," IEEE Trans. Pattern Anal. Mach. Intel., Vol. 11,July 1989 [7] J.M. Shapiro, "Embedded Image Coding Using Zerotrees of Wavelet Coefficients," IEEE Trans. Siganl Processing, Vol.41, No. 12, pp. 3445-3462, 1993.

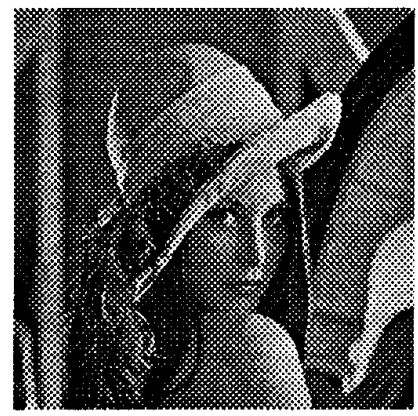

(a)

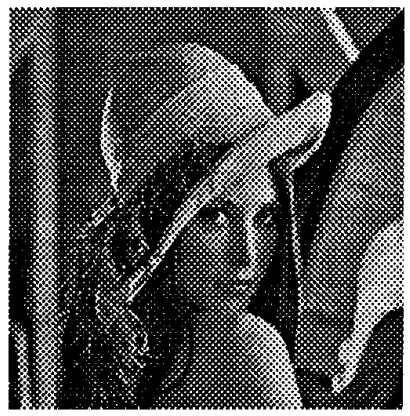

(b)

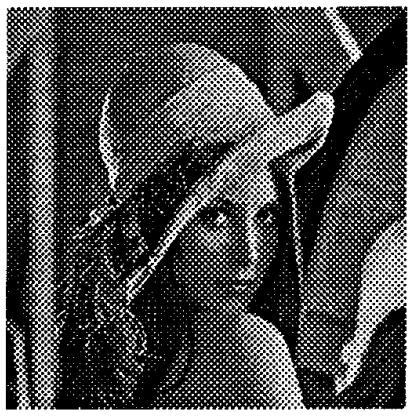

(c)

Fig.1 Reconstructed results for $512 \times 512$ image Lena

(a) original;

(b) PSNR: 34.5 at $0.4 \mathrm{bbp}$;

(c) PSNR: 32.3 at $0.2 \mathrm{bbp}$ 